\title{
Combination therapy of intravitreal bevacizumab with single simultaneous posterior subtenon triamcinolone acetonide for macular edema due to branch retinal vein occlusion \\ J Moon, M Kim and M Sagong
}

\author{
Abstract \\ Purpose To evaluate efficacy and safety of \\ combination therapy of intravitreal \\ bevacizumab (IVB) with single simultaneous \\ posterior subtenon triamcinolone acetonide \\ (STA) for treatment of macular edema (ME) \\ secondary to branch retinal vein occlusion \\ (BRVO). \\ Methods This was a prospective, randomized, \\ interventional comparative study conducted \\ in 45 eyes with ME secondary to BRVO \\ who were treated primarily with IVB $1.25 \mathrm{mg}$ \\ (23 eyes, IVB group) or combination therapy \\ of IVB $1.25 \mathrm{mg}$ with a single simultaneous \\ STA 40 mg (18 eyes, IVB/STA group). \\ Reinjections were performed with IVB if \\ optical coherence tomography (OCT) showed \\ recurrent $\mathrm{ME}$ associated with decreased visual \\ acuity. The main outcome measurement was \\ the number of additional IVB injections, \\ and changes of best-corrected visual acuity \\ (BCVA) and central macular thickness (CMT) \\ during a 6-month follow-up period were \\ compared. \\ Results BCVA showed significant \\ improvement in two groups at 6 months. \\ In addition, CMT showed significant decrease \\ in two groups at 6 months. No significant \\ differences in the change of BCVA and CMT \\ at 6 months after injection $(P=0.973$, \\ $P=0.639)$ were observed between the two \\ groups. A statistically significant difference \\ was found regarding the number of \\ additional IVB injections (IVB group \\ $0.96 \pm 0.83 ;$ IVB/STA group $0.44 \pm 0.70$, \\ $P=0.034)$.
}

Department of Ophthalmology, Yeungnam University College of Medicine, Daegu, South Korea

Correspondence: M Sagong, Department of Ophthalmology, Yeungnam University Medical Center, \#170 Hyeonchung-ro, Nam-gu, Daegu 705-802, South Korea

Tel: +82 53620 3443;

Fax: +82536265936.

E-mail: msagong@ynu.ac.kr

Received: 24 December 2015

Accepted in revised form: 23 March 2016

Published online:

27 May 2016
Conclusion Although combination therapy of IVB with a single simultaneous STA for treatment of ME secondary to BRVO did not affect the visual outcomes compared with IVB monotherapy, it had a benefit of reducing the number of additional IVB injections.

Eye (2016) 30, 1084-1090; doi:10.1038/eye.2016.96; published online 27 May 2016

\section{Introduction}

Branch retinal vein occlusion (BRVO) is the second most common retinal vascular disease after diabetic retinopathy. Macular edema (ME) is a common complication as a major cause of visual loss in BRVO patients. ${ }^{1,2}$ Although the exact mechanism of ME in BRVO is not fully understood, $\mathrm{ME}$ is thought to be caused by the flux of fluid from the blood vessels to the tissue, because of breakdown of the blood-retinal barrier as a result of damage to the tight junctions of capillary endothelial cells, enhanced expression of inflammatory cytokines such as prostaglandins and interleukin 6, and increased secretion into the vitreous of vasopermeability factors such as vascular endothelial growth factor (VEGF) produced in the retina. ${ }^{3-7}$

Several recent studies have reported that VEGF has an important role in the pathophysiology of ME by inducing blood-retinal barrier breakdown and increasing vascular permeability. ${ }^{6-9}$ Elevated VEGF level in vitreous has been reported in patients with BRVO, and this elevation was correlated with the severity of 
ME and area of non-perfusion. ${ }^{7}$ These correlations prompted interest in the use of pharmacologic inhibition of VEGF for treatment of ME. In several clinical studies bevacizumab, one of anti-VEGF agent, was reported to significantly reduce $\mathrm{ME}$ and improve visual function in BRVO. ${ }^{10-12}$

However, its half-life in vitreous is as short as 4.32 days and its effective concentration is maintained for 30 days, thus multiple injection is usually required for maintaining its effect. ${ }^{13}$ Multiple injections of bevacizumab increase the risk of injection-related complications such as vitreous hemorrhage, retinal detachment, and endophthalmitis, and it can be an economic burden to patients.

Because of its anti-inflammatory and anti-VEGF effects, ${ }^{14}$ corticosteroids have been reported to reduce $\mathrm{ME}$ and improve visual function in ME secondary to BRVO. ${ }^{15-18}$ Intravitreal triamcinolone acetonide (IVTA) has been found to be effective in reducing ME and improving visual function, but its use is limited by high risk of IOP elevation and cataract progression. ${ }^{15-17,19,20}$ Posterior subtenon triamcinolone acetonide (STA) has the advantage of easy injection and decreased risk of intraocular complications such as IOP elevation and cataract progression compared with IVTA, but the efficacy of STA is thought be slightly less than that of IVTA. ${ }^{21-23}$ Intravitreal dexamethasone implant has recently been found to be effective in reducing $\mathrm{ME}$ and improving visual function. In addition, its risk of complications such as glaucoma and cataract was relatively lower and its intraocular half-life was longer. ${ }^{18}$ However, due to its high cost, intravitreal dexamethasone implant can be a major economic burden for patients.

Each treatment has pros and cons. Thus combination therapy of corticosteroids and anti-VEGF has been attempted in clinical practice expecting a synergistic effect. ${ }^{24-27}$ Ehrlich et al ${ }^{24}$ reported that combining IVTA with IVB seems to offer no advantage over IVB alone for improving vision in BRVO, but it has the potential to reduce the frequency of repeated injections. And, to the best of our knowledge, no study evaluating the benefit of combination therapy of IVB with a single simultaneous STA compared with IVB monotherapy for treatment of ME secondary to BRVO has been reported, therefore, the aim of this study was to evaluate the benefit of combination therapy of IVB with a single simultaneous STA.

\section{Materials and methods}

This prospective interventional comparative study is a randomized, off-label, single-center investigation approved by the local institutional review board. Thus, its potential risks and benefits were discussed with the patients prior to the signing of an informed consent form.
In addition, this study was registered on June 5, 2012, at www.clinicaltrials.gov (NCT01614509).

Forty five eyes of 45 patients with ME secondary to BRVO (confirmed clinically and angiographically) from July 2012 to January 2013 were enrolled in the study. Patients who met the following criteria were included: (1) $\log$ MAR visual acuity $\geq 0.3$ (Snellen equivalent $\leq 20 / 40$ ), (2) ME secondary to BRVO, as confirmed by optical coherence tomography (OCT; Stratus, Carl Zeiss Meditec, Inc., Dublin, CA, USA). Exclusion criteria were: (1) other ocular vascular diseases causing ME (eg, central retinal vein occlusion, hypertensive retinopathy, and diabetic retinopathy); (2) treatment with focal laser photocoagulation or IVTA or IVB injection within the last 6 months; (3) history of intraocular surgery (including lens replacement surgery) within the last 6 months; (4) presence of comorbid ocular conditions that could affect VA.

Patients were divided into two groups with computergenerated randomization using Excel (Microsoft Excel 2010, SP1 MSO). Patients received one of the following procedures: IVB injection alone (IVB group; $n=24$ ), IVB with STA injection (IVB/STA group; $n=21$ ). All procedures were performed under aseptic conditions in the operating room. Topical anesthetic eye drops (proparacaine hydrochloride $0.5 \%$, Alcaine; Alcon Laboratories, Fort Worth, TX, USA) were instilled to the eye to be injected, followed by application of $5 \%$ povidone iodine to the periocular area, eyelids, eyelashes, and conjunctiva. Then the eye was opened using an eye speculum and bevacizumab (Avastin, Roche, Basel, Switzerland) $1.25 \mathrm{mg} / 0.05 \mathrm{ml}$ was injected through the pars plana $3.5 \mathrm{~mm}$ posterior to the limbus in phakic eyes and $3.0 \mathrm{~mm}$ posterior to the limbus in pseudophakic eyes using a 30-gauge needle. In the IVB/STA group, posterior subtenon triamcinolone acetonide injection was performed immediately after IVB injection. Forty milligrams $(1.0 \mathrm{ml})$ of triamcinolone acetonide (Triam, Shin poong pharm. Co., Ltd., Seoul, Korea) were injected slowly into the posterior subtenon space. Topical antibiotics of moxifloxacin (Vigamox; 0.5\% moxifloxacin hydrochloride ophthalmic solution, Alcon Laboratories, Inc., Fort Worth, TX, USA, no preservative) were prescribed four times daily for 3 days before and 4 days after all injections. Complete ophthalmic examinations including BCVA, tonometry, slit-lamp biomicroscopy fundus examination, and OCT were performed at baseline and monthly. If there persists ME on OCT after one initial injection, then we performed additional IVB injections monthly until complete resolution of ME is indicated on OCT. And then additional IVB injection was performed in the case of an increase in CMT $>50 \mu \mathrm{m}$ associated with decrease of BCVA during the follow-up period. 
The primary outcome was the number of additional IVB injections due to recurred ME during 6 months. The secondary outcome measures included changes in BCVA and CMT from baseline to 6 months, BCVA, and CMT at 1,3 and 6 months after injection, time point of additional IVB injection. In addition, safety profiles including adverse effects such as IOP elevation, retinal detachment, endophthalmitis, and vitreous hemorrhage were evaluated.

\section{Statistical analysis}

Statistical analysis was performed using SPSS software (PASW Statistics 18.0 for windows, SPSS Inc., Chicago, IL, USA). Mann-Whitney $U$-test was used for comparison of the mean change of BCVA and CMT, the number of additional IVB injections and IOP during 6 months between the two groups. Wilcoxon's signed-ranks test was used for comparison of preoperative and postoperative BCVA and CMT for each group. Fisher's Exact test was used to compare the number of additional IVB injections within 6 months from initial treatment between the two groups. $P$-values $<0.05$ were considered statistically significant.

\section{Results}

\section{Demographics of patients}

A total 45 eyes of 45 patients were included in this study (24 eyes in the IVB group and 21 eyes in the IVB/STA group). Four patients (one patient in the IVB group and three patients in the IVB/STA group) were dropped because of loss to follow-up, additional intraocular surgery, panretinal photocoagulation, or grid laser within 6 months or other ocular disease. Forty-one of 45 patients completed the 6-month follow-up (23/24 patients in the IVB group, 18/21 patients in the IVB/STA group). There was no statistically difference in age, gender, baseline BCVA, and baseline CMT between the two groups (Table 1).

Table 1 Baseline characteristics

\begin{tabular}{lccc}
\hline & IVB Group & IVB/STA Group & P-value \\
\hline Mean age (yrs) \pm SD & $60.57 \pm 10.68$ & $58.83 \pm 15.66$ & 0.415 \\
Female/male (No. of eyes) & $10 / 13$ & $9 / 9$ & 0.683 \\
Baseline BCVA (logMAR) & $0.60 \pm 0.24$ & $0.65 \pm 0.43$ & 0.642 \\
Baseline CMT $(\mu \mathrm{m})$ & $510.35 \pm 185.36$ & $468.22 \pm 159.26$ & 0.607 \\
\hline
\end{tabular}

Abbreviations: BCVA, best-corrected visual acuity; CMT, central macular thickness; IVB group, intravitreal bevacizumab injection alone; IVB/STA group, combines intravitreal bevacizumab with subtenon triamcinolone acetonide injection.

$P$-value: Mann-Whitney $U$-test.

\section{Comparison of injections between IVB and IVB/STA groups}

After initial treatment, ME recurred in 14 of 23 eyes $(60.9 \%)$ in the IVB group and 6 of 18 eyes $(33.3 \%)$ in the IVB/STA group. In the IVB group, 14 of 23 eyes $(60.9 \%)$ exhibiting recurrent $\mathrm{ME}$ received IVB reinjections. Among these 14 eyes, 8 eyes received one additional injection, 4 eyes received two additional injections, and 2 eyes received three additional injections. In the IVB/STA group, 6 of 18 eyes (33.3\%) exhibiting recurrent ME received IVB reinjections. Among these 6 eyes, 4 eyes received 1 additional injection, and 2 eyes received 2 additional injections. The mean number of additional IVB injections was $0.96 \pm 0.83$ in the IVB group and $0.44 \pm 0.70$ in the IVB/STA group during the follow-up period of 6 months, which was a significant difference between the two groups $(P=0.034$; Figure 1a).

Average time to first IVB reinjection was $2.07 \pm 0.73$ months in the IVB group, $3.00 \pm 1.10$ months in the IVB/STA group, but there was no significant difference between the two groups $(P=0.637)$. The number of eyes who had additional IVB due to recurrent ME within 2 months after initial injection was 12 of 23 eyes (52.2\%) in the IVB group and 2 of 18 eyes $(11.1 \%)$ in the IVB/STA group. Significant difference was observed between the two groups $(P<0.001$; Figure $1 b)$.

\section{Comparison of functional outcomes between IVB and IVB/STA groups}

In the IVB group, the mean BCVA increased from $\log$ MAR $0.60 \pm 0.24$ at baseline to $0.28 \pm 0.29$ at 6 months $(P=0.014)$. In the IVB/STA group, the mean BCVA increased from $\log$ MAR $0.65 \pm 0.43$ at baseline to $0.41 \pm 0.35$ at 6 months $(P=0.001$; Table 2, Figure $2 \mathrm{a})$. Mean BCVA improved significantly from baseline in both groups. No significant difference in mean BCVA at any follow-up was observed between the two groups.

In the IVB group, the mean change of BCVA from baseline was $\log$ MAR $0.18 \pm 0.19,0.16 \pm 0.23$, and $0.22 \pm 0.23$ at 1,3 and 6 months. In the IVB/STA group, the mean change of BCVA from baseline was logMAR $0.15 \pm 0.22,0.23 \pm 0.22$, and $0.25 \pm 0.22$ at 1,3 , and 6 months (Figure 2b). No significant difference in the change of BCVA at any time point was observed between the two groups.

\section{Comparison of anatomical outcomes between IVB and IVB/STA groups}

In the IVB group, the mean CMT decreased from $510.35 \pm 185.36 \mu \mathrm{m}$ at baseline to $246.48 \pm 88.00 \mu \mathrm{m}$ at 6 months $(P<0.001)$. In the IVB/STA group, the mean 
a

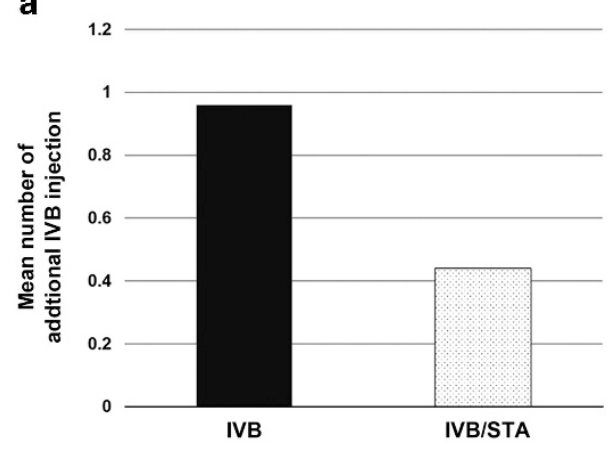

b

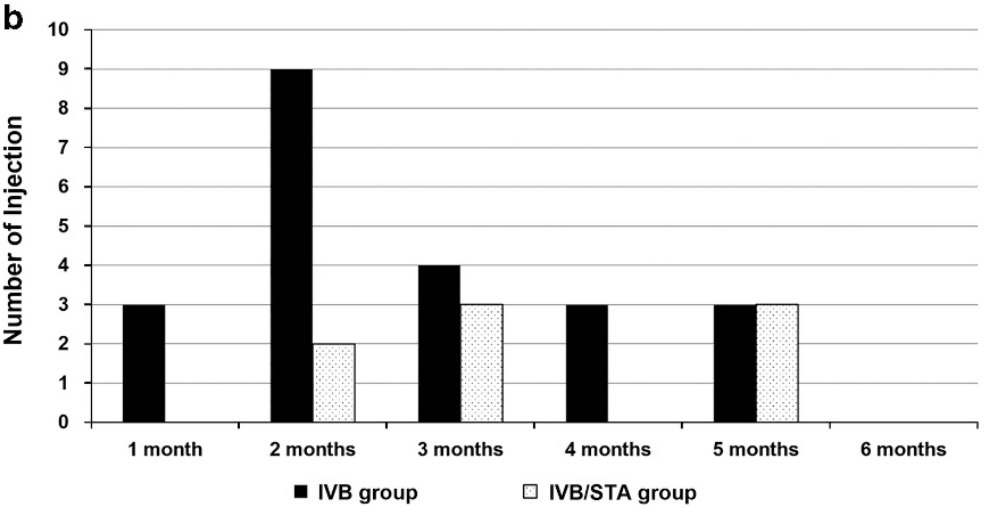

Figure 1 Comparison of reinjections between the two groups. (a) Mean number of additional IVB injections during follow-up periods between the two groups $(P=0.034)$. (b) Timing and number of reinjections during follow-up periods in the two groups.

Table 2 Changes of BCVA and CMT between the two groups

\begin{tabular}{|c|c|c|c|c|c|c|}
\hline & \multicolumn{3}{|c|}{$B C V A$} & \multicolumn{3}{|c|}{ CMT } \\
\hline & IVB group & IVB/STA group & P-value & IVB group & IVB/STA group & P-value \\
\hline Baseline & $0.60 \pm 0.24$ & $0.65 \pm 0.43$ & 0.642 & $510.35 \pm 185.36$ & $468.22 \pm 159.26$ & 0.607 \\
\hline 1 month & $0.42 \pm 0.24$ & $0.46 \pm 0.35$ & 0.961 & $291.48 \pm 100.19$ & $233.33 \pm 79.95$ & 0.015 \\
\hline 3 months & $0.44 \pm 0.28$ & $0.42 \pm 0.32$ & 0.615 & $265.35 \pm 106.85$ & $233.22 \pm 57.09$ & 0.742 \\
\hline 6 months & $0.38 \pm 0.29$ & $0.41 \pm 0.35$ & 0.973 & $246.48 \pm 88.00$ & $217.83 \pm 42.64$ & 0.639 \\
\hline
\end{tabular}

Abbreviations: BCVA, best-corrected visual acuity; CMT, central macular thickness; IVB group, intravitreal bevacizumab injection alone; IVB/STA group, combines intravitreal bevacizumab with subtenon triamcinolone acetonide injection.

$P$-value: Mann-Whitney $U$-test.

CMT decreased from $468.22 \pm 159.26 \mu \mathrm{m}$ at baseline to $217.83 \pm 42.64 \mu \mathrm{m}$ at 6 months (Table 2, Figure 2c). The CMT was significantly decreased in the IVB/STA group compared with the IVB group at 1 month $(P=0.015)$, but no significant difference with respect to CMT at 3 and 6 months was observed between the two groups $(P=0.742, P=0.639)$.

The mean change of CMT from baseline to 6 months was $263.87 \pm 204.19 \mu \mathrm{m}$ in the IVB group, and $250.39 \pm 159.13 \mu \mathrm{m}$ in the IVB/STA group. No significant difference in the change of CMT at any time point was observed between the two groups.

\section{Adverse events}

In the current study, none of the possible complications (endophthalmitis, retinal detachment, vitreous hemorrhage, and cataract progression) were observed in both groups. During the follow-up period, there was no IOP elevation requiring use of anti-glaucoma eye drops or performance of filtering surgery in both groups.

In two groups, the baseline IOP was $14.78 \pm 2.28 \mathrm{mmHg}$ in the IVB group vs $15.00 \pm 2.87 \mathrm{mmHg}$ in the IVB/STA group. At 6 months after initial injection, the IOP was $15.09 \pm 2.64 \mathrm{mmHg}$ in the IVB group and $15.56 \pm 3.03 \mathrm{mmHg}$ in the IVB/STA group. No significant difference was observed between the two groups at 1 and 6 months $(P=0.749, P=0.528)$.

\section{Discussion}

In this study, combination therapy of IVB with a single simultaneous STA did not have benefit functionally and anatomically compared with IVB alone for ME secondary to BRVO, however, combination therapy had benefit of reducing the number of additional IVB injections for recurrent ME.

The IVB/STA group showed significantly fewer additional IVB injections than the IVB group. This result indicates that therapeutic effects of combination therapy last longer than those of monotherapy; thus, combination therapy can delay or reduce recurrence of ME due to BRVO. We believe that it was due to the synergy of strong but short (30 days) anti-VEGF effect of IVB and weak but long (113 days) anti-inflammatory effect of STA. ${ }^{28}$ Comparing the periods of reinjection time, the IVB group showed $2.07 \pm 0.73$ months, the IVB/STA group showed $3.00 \pm 1.10$ months. Although there was no statistical difference between the two groups $(P=0.637)$, the difference of 1 month is meaningful time in the real-world 
a
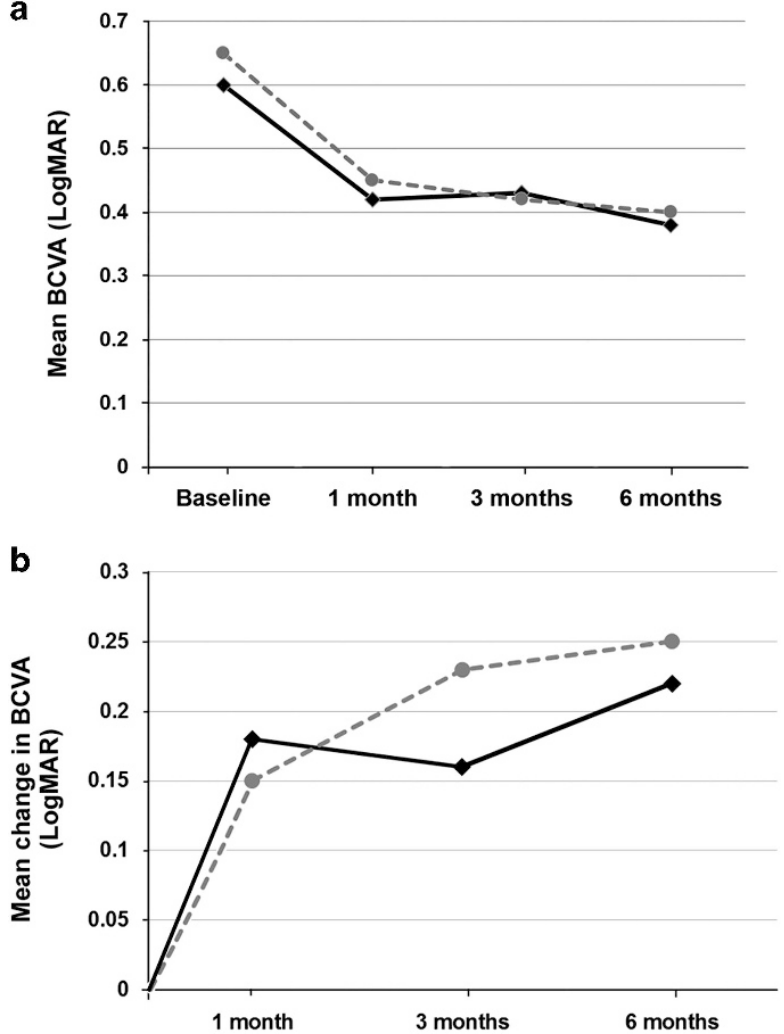

c

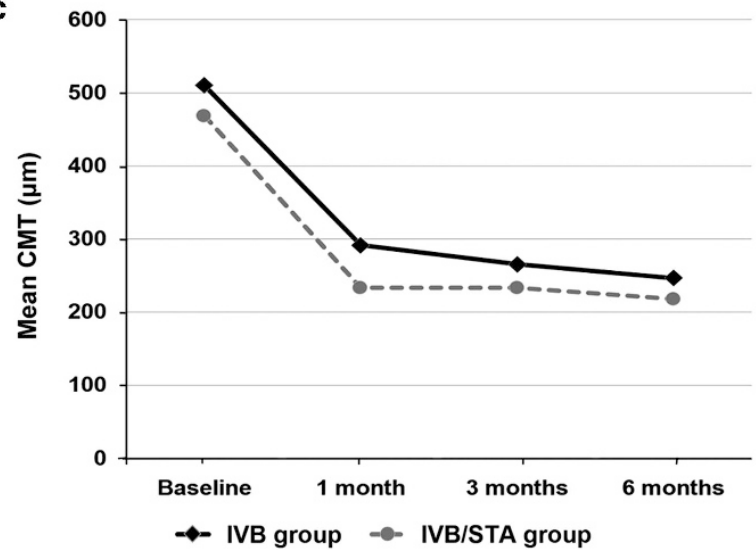

Figure 2 Comparison of functional and anatomical outcomes between the two groups. (a) The mean BCVA (logMAR) during follow-up periods between the two groups. At 1, 3 and 6 months after the initial injection, there was a significant improvement from baseline within this group (IVB; $P=0.018, P=0.041$, $P=0.014$, IVB $/$ STA; $P=0.012, P=0.002, P=0.001$ ). (b) The mean change in BCVA (logMAR) from baseline during follow-up periods between the two groups. There was a no significant difference at any time point between two groups. (c) The Mean central macular thickness (CMT) during follow-up periods between the two groups. At 1, 3 and 6 months after the initial injection, there was a significant improvement from baseline within this group (IVB and IVB/STA; $P<0.001, P<0.001$, $P<0.001)$. There was a significant difference between the two groups at 1 month $(P=0.015)$. setting. This result also indicates the incremental benefit of combination therapy, and it is in line with those of previous studies on combination therapy of corticosteroids and anti-VEGF agent. Ehrlich et al ${ }^{24}$ reported that combined treatment of IVB with IVTA has the potential to reduce the frequency of repeated injections. Maturi et al ${ }^{26}$ reported that combination therapy of IVB with dexamethasone implant requires fewer IVB reinjections than IVB monotherapy for treatment of ME due to RVO. Singer et al ${ }^{27}$ reported that combination of dexamethasone implant with IVB is synergistic in lengthening the time between injections as compared with either of these medications alone for treatment of ME due to RVO. Dexamethasone implant has a much stronger and relatively longer effect than STA, thus a direct comparison with this study using STA can be somewhat disputable; however, the synergistic effect of combination therapy of corticosteroids and anti-VEGF agent for treatment of ME due to RVO is indisputable.

In the mean change of BCVA after the first injection, there was no significant difference in any time points between the two groups. These results are in line with those of previous studies comparing IVB monotherapy with combination therapy. In one report comparing combination therapy of IVB and IVTA with IVB monotherapy, there was no advantage of combination therapy for improving vision at 6 months. ${ }^{24}$ In another study comparing combination therapy of IVB and dexamethasone implant with IVB monotherapy, mean changes in BCVA from baseline were similar in the two groups. ${ }^{26}$

The CMT was significantly decreased in the IVB/STA group compared with the IVB group at 1 month $(P=0.015)$, but there was no significant difference between the two groups at 3 and 6 months. These results are similar to those of previous studies comparing combination therapy of IVB and IVTA with IVB monotherapy..$^{24,25}$

This study showed no IOP elevation requiring use of anti-glaucoma eye drops or filtering surgery. Mean IOP elevation at 6 months after the first injection was only $0.31 \mathrm{mmHg}$ in the IVB group and $0.56 \mathrm{mmHg}$ in the IVB/STA group. In the previously reported study, the mean rise of IOP after a single injection of STA was $6.7 \mathrm{mmHg}{ }^{29}$ Also, incidence of IOP rise was lower in the STA than IVTA. ${ }^{22}$ Kawamura $e a^{23}$ reported that frequent injection of STA also resulted in IOP elevation. However, according to these studies, a single injection of STA might be a safe treatment modality in terms of IOP elevation.

In general, BRVO has a good prognosis: $50-60 \%$ of eyes have been reported to have a final VA of 20/40 or better even without treatment. ${ }^{30-32}$ Over a 1-year period, $5-15 \%$ of eyes developed ME, but of those with ME at baseline, $18-41 \%$ resolved. ${ }^{33}$ When considering this benign course 
of BRVO, it may be encouraging to many clinicians that combination therapy of IVB with STA results in reduction of the frequency of reinjection and injection-related complications showing efficacy similar to that of IVB monotherapy. The cost of treatment with STA is lower than that of an intravitreal dexamethasone implant or repeated IVB injection. ${ }^{34}$ Thus, the combination therapy of IVB with STA has safer profiles compared with IVTA, longer duration than IVB, and better cost effectiveness than dexamethasone implant.

Limitations of this study include a small population (45 patients) and short duration of follow-up (6 months). The long-term efficacy, safety, and the need for additional STA should be evaluated with long-term follow-up. In addition, visual acuity measured using Snellen charts has lower reproducibility of visual acuity measurements than ETDRS charts. Therefore further study using ETDRS charts with long-term follow-up is recommended.

In conclusion, although combination therapy of IVB with a single simultaneous STA for treatment of ME secondary to BRVO has no advantage to reducing ME and improving visual function compared with IVB alone, it had benefit of reducing the number of additional IVB injections. Therefore, combination therapy of IVB with a single simultaneous STA can be considered as a treatment options for ME secondary to BRVO.

\section{Summary}

What was known before

- There was no information about combination therapy of intravitreal bevacizumab and posterior subtenon triamcinolone acetonide for the treatment of macular edema due to branch retinal vein occluision.

What this study adds

- Combination therapy of intravitreal bevacizumab and posterior subtenon triamcinolone acetonide has advantages of less injections than intravitreal avastin alone, better safety than intravitreal triamcinolone alone, and economically more benefit than dexamethasone implant.

\section{Conflict of interest}

The authors declare no conflict of interest.

\section{Acknowledgements}

This study was funded by the 2015 Yeungnam University research grant.

\section{References}

1 Rogers S, McIntosh RL, Cheung N, Lim L, Wang JJ, Mitchell P et al. The prevalence of retinal vein occlusion: pooled data from population studies from the United States, Europe, Asia, and Australia. Ophthalmology 2010; 117(2): 313-319 e1.

2 Cugati S, Wang JJ, Rochtchina E, Mitchell P. Ten-year incidence of retinal vein occlusion in an older population: the Blue Mountains Eye Study. Arch Ophthalmol 2006; 124(5): 726-732.

3 Silva RM, Faria de Abreu JR, Cunha-Vaz JG. Blood-retina barrier in acute retinal branch vein occlusion. Graefes Arch Clin Exp Ophthalmol 1995; 233(11): 721-726.

4 Rehak J, Rehak M. Branch retinal vein occlusion: pathogenesis, visual prognosis, and treatment modalities. Curr Eye Res 2008; 33(2): 111-131.

5 Antonetti DA, Barber AJ, Khin S, Lieth E, Tarbell JM, Gardner TW. Vascular permeability in experimental diabetes is associated with reduced endothelial occludin content: vascular endothelial growth factor decreases occludin in retinal endothelial cells. Penn State Retina Research Group. Diabetes 1998; 47(12): 1953-1959.

6 Campochiaro PA, Hafiz G, Shah SM, Nguyen QD, Ying H, Do DV et al. Ranibizumab for macular edema due to retinal vein occlusions: implication of VEGF as a critical stimulator. Mol Ther 2008; 16(4): 791-799.

7 Noma H, Minamoto A, Funatsu H, Tsukamoto H, Nakano K, Yamashita $\mathrm{H}$ et al. Intravitreal levels of vascular endothelial growth factor and interleukin- 6 are correlated with macular edema in branch retinal vein occlusion. Graefes Arch Clin Exp Ophthalmol 2006; 244(3): 309-315.

8 Noma H, Funatsu H, Yamasaki M, Tsukamoto H, Mimura T, Sone $\mathrm{T}$ et al. Pathogenesis of macular edema with branch retinal vein occlusion and intraocular levels of vascular endothelial growth factor and interleukin-6. Am J Ophthalmol 2005; 140(2): 256-261.

9 Park SP, Ahn JK, Mun GH. Aqueous vascular endothelial growth factor levels are associated with serous macular detachment secondary to branch retinal vein occlusion. Retina 2010; 30(2): 281-286.

10 Rabena MD, Pieramici DJ, Castellarin AA, Nasir MA, Avery RL. Intravitreal bevacizumab (Avastin) in the treatment of macular edema secondary to branch retinal vein occlusion. Retina 2007; 27(4): 419-425.

11 Hikichi T, Higuchi M, Matsushita T, Kosaka S, Matsushita R, Takami K et al. Two-year outcomes of intravitreal bevacizumab therapy for macular oedema secondary to branch retinal vein occlusion. Br J Ophthalmol 2014; 98(2): 195-199.

12 Wu L, Arevalo JF, Roca JA, Maia M, Berrocal MH, Rodriguez FJ et al. Comparison of two doses of intravitreal bevacizumab (Avastin) for treatment of macular edema secondary to branch retinal vein occlusion: results from the Pan-American Collaborative Retina Study Group at 6 months of follow-up. Retina 2008; 28(2): 212-219.

13 Bakri SJ, Snyder MR, Reid JM, Pulido JS, Singh RJ. Pharmacokinetics of intravitreal bevacizumab (Avastin). Ophthalmology 2007; 114(5): 855-859.

14 McAllister IL, Vijayasekaran S, Chen SD, Yu DY. Effect of triamcinolone acetonide on vascular endothelial growth factor and occludin levels in branch retinal vein occlusion. Am J Ophthalmol 2009; 147(5): 838-846, 846.e1-2.

15 Scott IU, Ip MS, VanVeldhuisen PC, Oden NL, Blodi BA, Fisher $\mathrm{M}$ et al. A randomized trial comparing the efficacy and safety of intravitreal triamcinolone with standard care to treat vision loss associated with macular Edema secondary to branch retinal vein occlusion: the Standard Care vs 
Corticosteroid for Retinal Vein Occlusion (SCORE) study report 6. Arch Ophthalmol 2009; 127(9): 1115-1128.

16 Lee H, Shah GK. Intravitreal triamcinolone as primary treatment of cystoid macular edema secondary to branch retinal vein occlusion. Retina 2005; 25(5): 551-555.

17 Jonas JB, Akkoyun I, Kamppeter B, Kreissig I, Degenring RF. Branch retinal vein occlusion treated by intravitreal triamcinolone acetonide. Eye (Lond) 2005; 19(1): 65-71.

18 Haller JA, Bandello F, Belfort Jr R, Blumenkranz MS, Gillies M, Heier J et al. Randomized, sham-controlled trial of dexamethasone intravitreal implant in patients with macular edema due to retinal vein occlusion. Ophthalmology 2010; 117(6): 1134-1146 e3.

19 Wingate RJ, Beaumont PE. Intravitreal triamcinolone and elevated intraocular pressure. Aust N Z J Ophthalmol 1999; 27(6): 431-432.

20 Young S, Larkin G, Branley M, Lightman S. Safety and efficacy of intravitreal triamcinolone for cystoid macular oedema in uveitis. Clin Experiment Ophthalmol 2001; 29(1): 2-6.

21 Cardillo JA, Melo Jr LA, Costa RA, Skaf M, Belfort Jr R, Souza-Filho AA et al. Comparison of intravitreal versus posterior sub-Tenon's capsule injection of triamcinolone acetonide for diffuse diabetic macular edema. Ophthalmology 2005; 112(9): 1557-1563.

22 Hayashi K, Hayashi H. Intravitreal versus retrobulbar injections of triamcinolone for macular edema associated with branch retinal vein occlusion. Am J Ophthalmol 2005; 139(6): 972-982.

23 Kawamura R, Inoue M, Shinoda H, Shinoda K, Itoh $\mathrm{Y}$, Ishida $\mathrm{S}$ et al. Incidence of increased intraocular pressure after subtenon injection of triamcinolone acetonide. J Ocul Pharmacol Ther 2011; 27(3): 299-304.

24 Ehrlich R, Ciulla TA, Moss AM, Harris A. Combined treatment of intravitreal bevacizumab and intravitreal triamcinolone in patients with retinal vein occlusion: 6 months of follow-up. Graefes Arch Clin Exp Ophthalmol 2010; 248(3): 375-380.

25 Cekic O, Cakir M, Yazici AT, Alagöz N, Bozkurt E, Faruk Yilmaz O. A comparison of three different intravitreal treatment modalities of macular edema due to branch retinal vein occlusion. Curr Eye Res 2010; 35(10): 925-929.

26 Maturi RK, Chen V, Raghinaru D, Bleau L, Stewart MW. A 6-month, subject-masked, randomized controlled study to assess efficacy of dexamethasone as an adjunct to bevacizumab compared with bevacizumab alone in the treatment of patients with macular edema due to central or branch retinal vein occlusion. Clin Ophthalmol 2014; 8: 1057-1064.

27 Singer MA, Bell DJ, Woods P, Pollard J, Boord T, Herro A et al. Effect of combination therapy with bevacizumab and dexamethasone intravitreal implant in patients with retinal vein occlusion. Retina 2012; 32(7): 1289-1294.

28 Shen L, You Y, Sun S, Chen Y, Qu J, Cheng L. Intraocular and systemic pharmacokinetics of triamcinolone acetonide after a single 40-mg posterior subtenon application. Ophthalmology 2010; 117(12): 2365-2371.

29 Iwao K, Inatani M, Kawaji T, Koga T, Mawatari Y, Tanihara H. Frequency and risk factors for intraocular pressure elevation after posterior sub-Tenon capsule triamcinolone acetonide injection. J Glaucoma 2007; 16(2): 251-256.

30 Gutman FA, Zegarra $\mathrm{H}$. The natural course of temporal retinal branch vein occlusion. Trans Am Acad Ophthalmol Otolaryngol 1974; 78(2): OP178-OP192.

31 Hayreh SS, Rojas P, Podhajsky P, Montague P, Woolson RF. Ocular neovascularization with retinal vascular occlusion-III. Incidence of ocular neovascularization with retinal vein occlusion. Ophthalmology 1983; 90(5): 488-506.

32 Magargal LE, Kimmel AS, Sanborn GE, Annesley WH Jr. Temporal branch retinal vein obstruction: a review. Ophthalmic Surg 1986; 17(4): 240-246.

33 Rogers SL, McIntosh RL, Lim L, Mitchell P, Cheung N, Kowalski JW et al. Natural history of branch retinal vein occlusion: an evidence-based systematic review. Ophthalmology 2010; 117(6): 1094-1101 e5.

34 Smiddy WE. Economic considerations of macular edema therapies. Ophthalmology 2011; 118(9): 1827-1833. 